\title{
ENSAIO DE IMPACTO IZOD EM MATRIX EPOXÍDICA REFORÇADA COM TECIDO DE FIQUE*
}

\author{
Maria Carolina Andrade Teles ${ }^{1}$ \\ Marcos Vinícius Fonseca Ferreira ${ }^{1}$ \\ Gilson Vieira Fernandes ${ }^{1}$ \\ Frederico Muylaert Margem ${ }^{2}$ \\ Sergio Neves Monteiro ${ }^{3}$ \\ Felipe Perissé Duarte Lopes ${ }^{4}$
}

\section{Resumo}

Questões socioambientais têm maximizado a permuta entre o uso de materiais sintéticos para o uso de materiais naturais. A fibra de fique disposta no presente trabalho na forma de tecido, extraída do folha da planta do fique (Furcraea Andina) demonstra grande potencialidade como reforço de compósito polimérico. Nos corpos de prova para os ensaios, as camadas de tecido foram misturadas à resina epoxídica DGEBA/TETA e justapostas sob pressão em um molde retangular metálico à temperatura ambiente por 24 horas. A quantidade de tecido de fique nos corpos de prova gerados, foram de até 5 camadas, cortados a partir das dimensões matriciais, seguindo a norma ASTM D256. Testados e após analisados por microscopia de varredura eletrônica (MEV). Pode-se notar o aumento significante da resistência ao impacto de acordo com o crescimento numérico de camadas fibrosas que reforçaram o compósito. Tal performance pode ser associada ao mecanismo de fratura, gerada da interação entre o tecido de fique e a matriz.

Palavras-chave: Impacto Izod; Tecido de Fique; Compósitos Epoxídicos; MEV.

\section{IZOD IMPACT TESTS IN EPOXI MATRIX COMPOSITES REINFORCED WITH FIQUE FABRIC}

\section{Abstract}

Social and environmental reasons have maximized the changes between use of synthetic materials by natural materials. The fique fiber disposed in the present work in the form of fabric, extracted from the leaf of the plant (Furcraea Andina) demonstrates a great potential as reinforcement of polymeric composite. For the tests, the fabric layers were mixed with the DGEBA / TETA epoxy resin and juxtaposed under pressure in a rectangular metal mold at room temperature for 24 hours. The amount of fique fabric were up to 5 layers, cut from the matrix dimensions the specimens follows the ASTM D256 standard. Tested and analysed by scanning electron microscopy (SEM). One note is the significant increase of impact strength according to numerical growth of fibrous layers into the composites. Such performance may be associated with the damage mechanism, generated from the interaction between the fique fabric and the matrix.

Keywords: Izod Impact; Fique Fabric; Epoxi Composites; SEM.

1 Graduando(a) em Engenharia Metalúrgica e de Materiais, bolsista de iniciação científica, LAMAV, UENF, Campos dos Goytacazes, Rio de Janeiro - Brasil.

2 Engenheiro de Produção, Doutor em Ciências e Engenharia de Materiais, Pós-Doutorando, LAMAV, UENF, Campos dos Goytacazes, Rio de Janeiro - Brasil.

3 Engenheiro Metalúrgico, Doutor em Ciência e Engenharia de Materiais, Professor titular, IME, Rio de Janeiro, Rio de Janeiro - Brasil.

4 Engenheiro de Metalúrgico, Doutor em Ciências de Materiais, Livre-Docência, LAMAV, UENF, Campos dos Goytacazes, Rio de Janeiro - Brasil. 


\section{INTRODUÇÃO}

As fibras à base de celulose estão sendo usadas devido à sua imagem "verde", de acordo com Kalia, Kaith e Kaurs [1]. Em vez das fibras de vidro, amplamente utilizadas na tecnologia moderna, as fibras lignocelulósicas são renováveis e podem ser incineradas no final da vida útil do material sem a adição de poluição do ar, uma vez que a quantidade de $\mathrm{CO}_{2}$ liberada durante a incineração é insignificante em relação à quantidade absorvida pela planta ao longo de sua vida.

Mesmo se 0 processamento industrial de liberação equivalente de $\mathrm{CO}_{2}$ for adicionada, a fibra lignocelulósica pode ser considerada neutra em relação à emissão de gases responsáveis pelo aquecimento global [2].

Aspectos ambientais estão relacionados ao fato de que as fibras naturais são renováveis, recicláveis e biodegradáveis. Além disso, seus compósitos exigem gasto energético comparativamente menor para serem processados. Estes aspectos combinados resultam na neutralidade do dióxido de carbono da fibra natural em contraste com as fibras sintéticas que contribuem para o aquecimento global através da sua emissão positiva de $\mathrm{CO}_{2}$ [3-5]. A vantagem econômica das fibras lignocelulósicas são seus preços significativamente mais baixos em relação à fibra de vidro, que é a mais barata entre as fibras sintéticas.

Os aspectos técnicos também podem ser favoráveis às fibras naturais que são mais suaves e, consequentemente, menos abrasivas aos equipamentos de processamento. Além disso, para algumas fibras lignocelulósicas, as propriedades mecânicas podem ser superiores à correspondente de compósitos de fibra de vidro $[4,5]$. Este é o caso particular das fibras lignocelulósicas que receberam um tratamento superficial para melhorar a adesão a uma matriz polimérica [5].

As fibras naturais também apresentam inconvenientes em relação ao uso como reforço composto. A alta adsorção de umidade na superfície devido à característica hidrofílica da fibra causa incompatibilidade com matrizes poliméricas hidrofóbicas [6]. Trabalhos anteriores demonstram que a incorporação de fibras lignocelulósicas na matriz polimérica dá origem a compósitos com resistência mecânica diretamente proporcional ao teor de fibras, onde essas fibras atuam como reforço para a matriz devido às suas altas propriedades mecânicas. Objetivando aumentar ainda mais as propriedades mecânicas oferecidas por tais compósitos, é avaliado a substituição de compósitos reforçados com fibras contínuas e alinhadas de fique pelo tecido de fique em testes de impacto izod.

\section{MATERIAIS E MÉTODOS}

Fornecido por um produtor da Colômbia, o material básico utilizado neste trabalho foi a fibra de fique, extraída da folha da planta de fique (Furcraea Andina) e organizada em forma de tecido de fique (Fig. 1). 
Tabela 1. Energia de Impacto Izod para composições epoxídicas reforçadas com tecido de fique

\begin{tabular}{c|c}
\hline Quantidade de Camadas de Tecido & Energy (J/m) \\
\hline 0 & $23.8 \pm 1.2$ \\
\hline 3 & $83.87 \pm 33.8$ \\
\hline 4 & $150.9 \pm 31.1$ \\
\hline 5 & $276.8 \pm 46.7$ \\
\hline
\end{tabular}

Baseado nos resultados demonstrados na Tabela 1, é apresentada a variação de Energia de Impacto Izod relacionada com a quantidade de camadas de tecido de fique em matriz epoxídica através da Fig. 3. Esta figura mostra um aumento significativo na energia de impacto Izod com o aumento da quantidade de camadas de tecido de fique. Mesmo considerando as barras de erro, é possível interpretar o aumento da energia de impacto como seguindo uma relação linear.

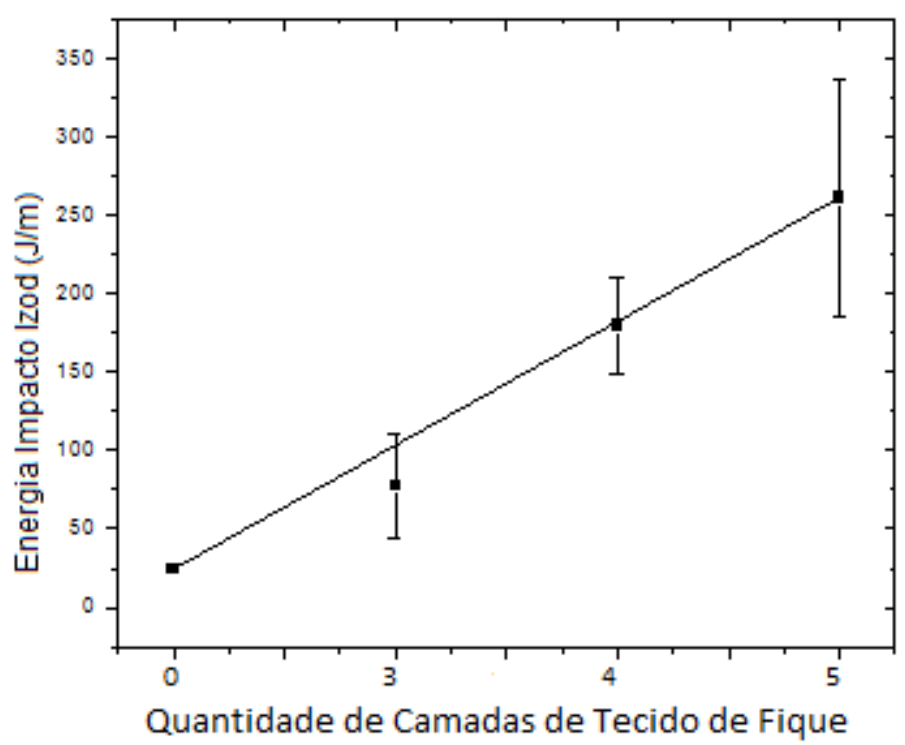

Figura 3. Energia de Impacto Izod em função da quantidade de camadas de tecido de Fique.

Os valores apresentados na Fig. 3 são consistentes com os resultados relatados na literatura. O reforço de uma matriz polimérica com fibras sintéticas e naturais [7-10] aumenta a resistência ao impacto do compósito. Assim, os compósitos absorvem quantidades de energia relativamente maiores, levando a um aumento da resistência ao impacto.

O fato dos tecidos permanecerem intactos após o impacto, como mostrado para os compósitos na Fig. 4, é uma indicação de que as trincas se propagaram ao longo da interface tecido / matriz, causando a separação do tecido do epóxi, mas não a ruptura do tecido. Esse efeito aumenta a trajetória das trincas através do composto, criando uma maior energia de impacto. 


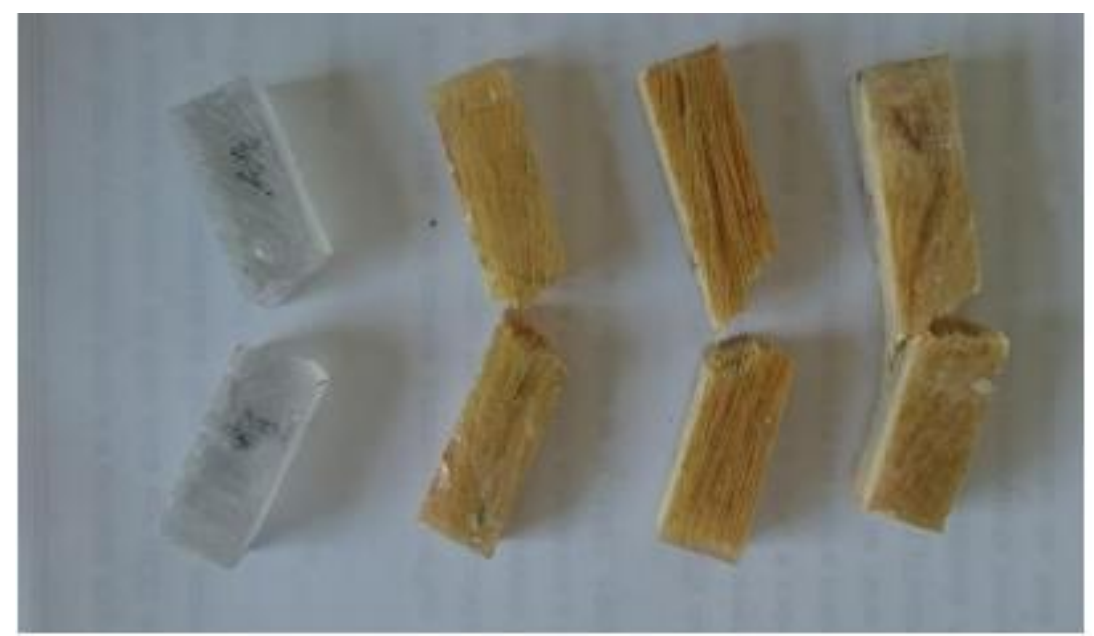

Figura 4. Espécies típicas de compósitos epoxídicos com diferentes quantidades de camadas de tecido de fique, fraturados a partir de Impacto Izod.

O corpo de prova com 5 camadas de tecido fique não foi completamente fraturado após o impacto. Isto é devido à resistência, relativamente alta, do tecido de fique, o que evita um colapso total. Para uma maior quantidade de camadas, o corpo de prova ensaiado foi curvado o suficiente para permitir que o martelo continuasse sua trajetória, enquanto as duas partes da amostra dobrada permanecessem atreladas. A não ocorrência de ruptura após o impacto indica uma alta resistência do compósito. Foi assumido que, se a ruptura tivesse ocorrido completamente, a energia absorvida seria ainda maior.

A análise microestrutural realizada pelo MEV da região dos corpos de provas fraturada no impacto Izod permitiu um melhor entendimento dos mecanismos responsáveis pela alta resistência dos compósitos reforçados com tecido de fique.

A Fig. 5 apresenta a fotografia tirada pelo MEV da superfície fraturada pelo teste de impacto Izod para as espécies epoxídicas reforçadas com 5 camadas de tecido de fique.

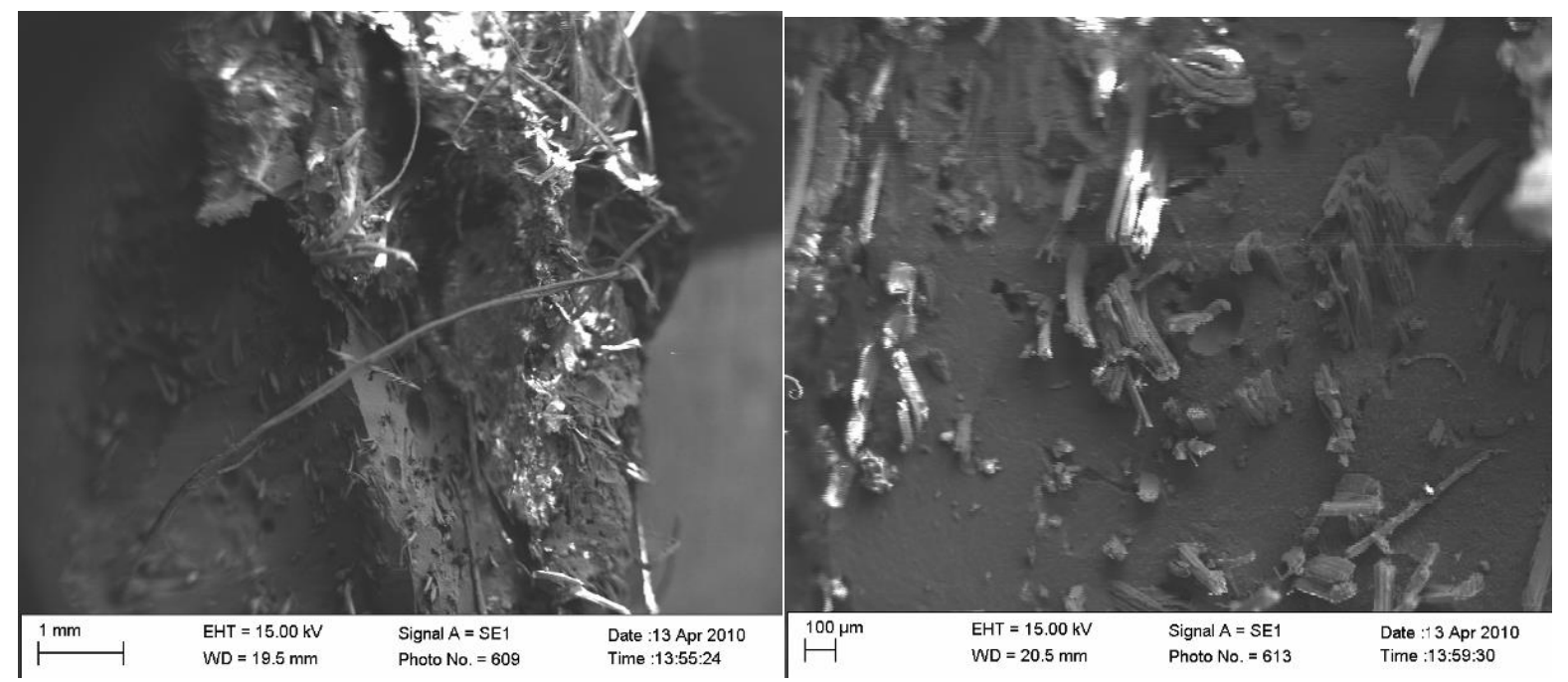

Figura 5. Micrografia MEV obtida da fratura dos compósitos reforçados com 5 camadas de tecido de fique em matriz epóxi. 
Nesta figura, pode ser notado com uma menor ampliação (Fig. 5a), a fratura da matriz epoxídica com as fibras incorporadas. Com uma ampliação maior (Fig. 5b) podem ser vistas evidências do tecido sendo puxado da matriz. Tal fato demonstra ser consequência da fraca adesão entre o tecido e a matriz que, quando exposto à um estresse de impacto, desliza um pouco e rompe quando não é mais possível deslizar.

\section{CONCLUSÃO}

- Os compósitos de matriz epoxídica reforçado com tecido de fique mostra uma melhora na resistência em comparação com a matriz de epóxi puro.

- A matriz epóxi incorporada com 5 camadas de tecido de fique melhora significativamente a resitência do composito, $276.8 \mathrm{~J} / \mathrm{m}$, em comparação com a resina epóxi pura, com $23.8 \mathrm{~J} / \mathrm{m}$.

- Grande parte desse aumento de é, aparentemente, devido à baixa resistência de cisalhamento interfacial do tecido / matriz epoxi tensionada. Isso resulta em maior energia absorvida como resultado da propagação de fissuras longitudinais ao longo da interface, o que leva a maiores áreas de ruptura em comparação com uma fratura transversal.

\section{Agradecimentos}

Os autores agradecem o apoio a esta investigação pelas agências brasileiras: CNPq, CAPES e FAPERJ.

\section{REFERÊNCIAS}

1 Kalia S, Kaith BS, Kaurs I. Cellulose Fibers: Bio - and Nano - Polymer Composites (New York: Springer, 2011).

2 Gore A. An Inconvenient Truth. The Planetary Emergency of Global Warming and What We Can do About It (Emmaus, Pennsylvania, USA: Rodale Press, 2006).

3 Zah R, Hischier R, Leão and I. Braun. Curaua fibers in the automobile industry - A sustainability assessment. J. Cleaner Production, 15 (2007) 1032-1040.

4 Wambua P, Ivens I, Verpoest I. Natural fibers: can they repalce glass and fibre reinforced plastics?, Composites Science and Technology, 63 (2003) 1259-1264.

5 Mohanty AK, Misra M, Hinrichsen G. Biofibers, biodegradable polymers and biocomposites: an overview, Macromolecular Mat. And Engineering, 276/277 (2000), 124.

6 Vasquez A, Riccieri J, Carvalho L. Interfacial properties and initial step of water sorption in uniderectional unsaturated polyester/vegetable fibre composites. Polymer Composites, 20 (1999) 29-27.

7 Leão AL, Tan IH, Caraschi JC. Curaua fiber - A tropical natural fibre from Amazon Potential and applications in composites, Proceedings of the International Conference on Advanced Composites, (Hurghada, Egypt, May, 1998) 557-564.

8 Monteiro SN, Aquino RCMP, Lopes FPD, Carvalho EA, d'Almeida, JRM. Charpy impact notch toughness of piassava fibers reinforced polyester matrix composites. (in Portuguese), Rev. Mater., 11(3) (2006) 204-210.

9 Fu SY, Lauke B, Mäder E, Hu X, Yue CY. Fracture resistance of short-glass-fiberreinforced and short-carbon-fiber-reinforced poly-propylene under charpy impact load and dependence on processing, J. Mater. Process. Technol., 89/90 (1999), 501-507. 
10 MONTEIRO SN, PEREIRA AC, INÁCIO WP. Izod impact energy of polyester matrix composites reinforced with aligned sisal fibers. In: Associação Brasileira de Metalurgia e Materiais - ABM, São Paulo, SP, Julho, 2011, p. 1-12. 